\title{
On the oscillation of second order non-linear differential equations
}

\author{
Hishyar Kh. Abdullah
}

Dept. of Mathematics, College of Science, University of Sharjah, P.O. Box 27272, Sharjah, U.A.E.

Email: hishyar@sharjah.ac.ae

Copyright (C)2014 Authors. This is an open access article distributed under the Creative Commons Attribution License, which permits unrestricted use, distribution, and reproduction in any medium, provided the original work is properly cited.

\begin{abstract}
In this paper we are concerned with the oscillation of second order non-linear homogeneous differential equation. An example has been given to illustrate the results.
\end{abstract}

Keywords: Oscillatory, Second order differential equations, Non-Linear.

\section{Introduction}

In this paper we are concerned with the oscillation of the second order non-linear differential equation of the form

$x^{\prime \prime}(t)+\left(x^{\prime}(t)\right)^{2}+a(t) f(x(t))=0$,

where $a(t)$ is a continuous real valued function on the interval $[\alpha, \infty)$, without any restriction on its sign and $\alpha \geq 0$ is a fixed non-negative real number. $f(x(t))$ is continuously differentiable functions on $R-\{0\}$ where $y f(y)>0$ and $f^{\prime}(y)>0$ for all $y(t) \neq 0$ and the following conditions holds for $f(y)$

$\infty \frac{\sqrt{\left(f(y)+f^{\prime}(y)\right)^{\prime}}}{f(y)+f^{\prime}(y)} d y<\infty$ and $-\infty \frac{\sqrt{\left(f(y)+f^{\prime}(y)\right)^{\prime}}}{f(y)+f^{\prime}(y)} d y<\infty$

and

$\min \left\{\inf _{y>0} \frac{\left[{ }_{y}^{\infty} \frac{\sqrt{\left(f(z)+f^{\prime}(z)\right)^{\prime}}}{f(z)+f^{\prime}(z)} d z\right]^{2}}{y_{y}^{\infty} \frac{d z}{\left(f(z)+f^{\prime}(z)\right)^{\prime}}} ; \inf _{y<0} \frac{\left[-\infty \frac{\sqrt{\left(f(z)+f^{\prime}(z)^{\prime}\right.}}{f(z)+f^{\prime}(z)} d z\right]^{2}}{y_{y}^{\infty} \frac{d z}{\left(f(z)+f^{\prime}(z)\right)^{\prime}}}\right\}>0$

Our attention is concentrated only to such solution $x(t)$ of the differential equation (1.1) which exists on some interval $[\beta, \infty)$, for $\beta \geq \alpha$.

Definition 1.1 A solution $x(t)$ of the differential equation (1.1) is said to be "nontrivial" if $x(t) \neq 0$ for at least one $t \in[\alpha, \infty)$. 
Definition 1.2 A nontrivial solution $x(t)$ of differential equation (1.1) is said to be oscillatory if it has arbitrarily large zeros on $[\beta, \infty)$, for $\beta>\alpha$ otherwise it said to be " non oscillatory.

Definition 1.3 The differential equation (1.1) is said to be oscillatory if a nontrivial solution $x(t)$ is oscillatory.

The study of the oscillation of a second order nonlinear ordinary differential equations is of special interest. Many criteria have been found which involve the behavior of the integral of a combination of the coefficients of second order nonlinear differential equations. This approach has been motivated by authors (for example see [1], $[2],[3],[4],[5],[6],[7],[8]$ and $[9]$ and the authors therein).

The purpose of this paper is to present new criteria of oscillation of the differential equation (1.1).

\section{Main results}

We prove the following theorem

Theorem 2.1 The differential equation (1.1) is oscillatory if

$\lim _{t \rightarrow \infty} \inf \int_{\alpha}^{t} a(s) d s>-\infty$

$\lim _{t \rightarrow \infty} \sup \frac{1}{t} \int_{\alpha}^{t}(t-s) a(s) d s=\infty$

and

$\lim _{t \rightarrow \infty} \sup \frac{1}{t^{n-1}} \int_{\alpha}^{t}(t-s)^{n-1} a(s) d s=\infty$ for some integer $n>2$,

where $f(x(t))$ satisfies the conditions (1.2) and (1.3) .

Let $x(t)$ be a nonoscillatory solution of $(1.1)$ on the interval $[\beta, \infty)$, where $\beta \geq \max \{\alpha, 1\}$, without loss of generality its solution can be supposed such that $x(t)>0$ on $[\beta, \infty)$.

We define

$w(t)=x^{\prime}(t) f^{-1}(x(t))$

Then $w(t)$ is well defined and satisfies the equation

$w^{\prime}(t)=-\left(f(x(t))+f^{\prime}(x(t))\right) w^{2}(t)-a(t)$

Integrating both sides of the above equation from $\beta$ to $t$ we get

$-w(t)=-w(\beta)+\int_{\beta}^{t} a(s) d s+\int_{\beta}^{t}\left(f(x(s))+f^{\prime}(x(s))\right) w^{2}(s) d s$.

We will consider two cases where

$\int_{\beta}^{\infty}\left(f(x(s))+f^{\prime}(x(s))\right) w^{2}(s) d s$ is finite or infinite.

Case I : If $\int_{\beta}^{\infty}\left(f(x(s))+f^{\prime}(x(s))\right) w^{2}(s) d s<\infty$.In this case we

$\int_{\beta}^{\infty}\left(f(x(s))+f^{\prime}(x(s))\right) w^{2}(s) d s \leq N$ for $t \geq \beta$ 
Where $N$ is a positive constant. Using Schwarz inequality for $t \geq \beta$ we derive

$$
\begin{aligned}
\left|\int_{\beta}^{t} w(s) \sqrt{f(x(s))+f^{\prime}(x(s))} d s\right|^{2} & \leq\left(\int_{\beta}^{t} d s\right) \int_{\beta}^{t} w^{2}(s)\left(f(x(s))+f^{\prime}(x(s))\right) d s \\
& =(t-\beta) \int_{\beta}^{t} w^{2}(s)\left(f(x(s))+f^{\prime}(x(s))\right) d s .
\end{aligned}
$$

Now using (2.6) we have

$$
\left|\int_{\beta}^{t} w(s) \sqrt{f(x(s))+f^{\prime}(x(s))} d s\right|^{2} \leq N t \text { for all } t \geq \beta
$$

Then by condition (2.1) there exist a constant $M>0$ such that

$\sqrt{f(x(t))+f^{\prime}(x(t))} \int_{y(t)}^{\infty} \frac{\sqrt{f(z)+f^{\prime}(z)}}{f(z)} d z \geq M$ for $t \geq \beta$.

Now put $K=\int_{y(t)}^{\infty} \frac{\sqrt{f(z)+f^{\prime}(z)}}{f(z)} d z>0$. Then in view of (2.7) and (2.8) for any $t \geq \beta$ we get

$$
\begin{aligned}
\left(f(x(t))+f^{\prime}(x(t))\right) & \geq M^{2}\left[\int_{y(t)}^{\infty} \frac{\sqrt{f(z)+f^{\prime}(z)}}{f(z)} d z\right]^{-2} \\
& =M^{2}\left[K-\int_{y(\beta)}^{y(t)} \frac{\sqrt{f(z)+f^{\prime}(z)}}{f(z)} d z\right]^{-2} \\
& =M^{2}\left[K-\int_{\beta}^{t} \frac{x^{\prime}(s)}{f(x(s))} \sqrt{f(x(s))+f^{\prime}(x(s))} d s\right]^{-2} \\
& \geq M^{2}\left[K+\int_{\beta}^{t} w(s) \sqrt{f(x(s))+f^{\prime}(x(s))} d s\right]^{-2} \\
& \geq M^{2}(K+\sqrt{N t})^{-2} \geq M^{2}(K \sqrt{t}+\sqrt{N t})^{-2} \\
& =\frac{M^{2}}{t}(K+\sqrt{N})^{-2} .
\end{aligned}
$$

Thus by setting

$C=\frac{M^{2}}{t}(K+\sqrt{N})^{-2}>0$,

we have

$\sqrt{f(x(t))+f^{\prime}(x(t))} \geq \frac{C}{t}$.

From (2.4) we get

$a(t)=w^{\prime}(t)-\left(f(x(t))+f^{\prime}(x(t))\right) w^{2}(t) \leq w^{\prime}(t)-\frac{C}{t} w^{2}(t)$, for every $t \geq \beta$.

Hence we obtain 


$$
\begin{aligned}
\int_{\beta}^{t}(t-s)^{n-1} a(s) d s \leq & \int_{\beta}^{t}(t-s)^{n-1} w^{\prime}(s) d s-C \int_{\beta}^{t} \frac{(t-s)^{n-1}}{s} w^{2}(s) d s \\
= & (t-\beta)^{n-1} w(\beta)-(n-1) \int_{\beta}^{t}(t-s)^{n-2} w(s) \\
-C \int_{\beta}^{t} \frac{(t-s)^{n-1}}{s} w^{2}(s) d s= & (t-\beta)^{n-1} w(\beta)-\frac{(n-1)^{n-2}}{4 C} \int_{\beta}^{t} s(t-s)^{n-3} d s \\
& -\int_{\beta}^{t}\left[\sqrt{\frac{C}{s}}(t-s)^{\frac{n-1}{2}} w(s)+\frac{n-1}{2} \sqrt{\frac{s}{C}}(t-s)^{\frac{n-3}{2}}\right]^{2} d s \\
\leq & (t-\beta)^{n-1} w(\beta)+\frac{(n-1)^{n-2}}{4 C} \int_{0}^{t-\beta}(t-u)^{n-3} d u \\
= & {\left[w(\beta)-\frac{n-1}{4 C}\right](t-\beta)^{n-1}+\frac{(n-1)^{2}}{4 C(n-2)} t(t-\beta)^{n-2} . }
\end{aligned}
$$

So that we have

$$
\begin{aligned}
\frac{1}{t^{n-1}} \int_{\beta}^{t}(t-s)^{n-1} a(s) d s \leq & \frac{1}{t^{n-1}} \int_{\beta}^{t}(t-s)^{n-1}|a(s)| d s+\frac{1}{t^{n-1}} \int_{\beta}^{t}(t-s)^{n-1} a(s) d s \\
\leq & \left(1-\frac{t_{0}}{t}\right)^{n-1} \int_{t_{0}}^{t}|a(s)| d s+\left[w(\beta)-\frac{n-1}{4 C}\right]\left(1-\frac{\beta}{t}\right)^{n-1} \\
& +\frac{(n-1)^{2}}{4 C(n-2)}\left(1-\frac{\beta}{t}\right)^{n-2}
\end{aligned}
$$

for all $t \geq \beta$. This gives

$\lim _{t \rightarrow \infty} \sup \frac{1}{t^{n-1}} \int_{\alpha}^{t}(t-s)^{n-1} a(s) d s \leq \int_{t_{0}}^{t}|a(s)| d s+w(\beta)-\frac{n-1}{4 C}+\frac{(n-1)^{2}}{4 C(n-2)}<\infty$.

Which contradicts the condition (2.3).

Case II : Suppose $\int_{\beta}^{\infty} w^{2}(s)\left(f(x(s))+f^{\prime}(x(s))\right)^{\prime} d s=\infty$.

By condition (2.3) from (2.5) it follows that there exist a constant $\mu$ such that

$-w(t) \geq \mu+\int_{\beta}^{t} w^{2}(s)\left(f(x(s))+f^{\prime}(x(s))\right) d s$, for all $t \geq \beta$.

We consider $\delta>\beta$ such that

$A=\mu+\int_{\beta}^{\delta} w^{2}(s)\left(f(x(s))+f^{\prime}(x(s))\right)^{\prime} d s>0$.

Then (2.9) says that $w(t)$ is negative on $[\delta, \infty]$,now multiplying (2.9) through by

$$
\frac{-w(t)\left(\left(f(x(t))+f^{\prime}(x(t))\right)^{\prime}\right.}{\left[\mu+\int_{\beta}^{\infty} w^{2}(s)\left(f(x(s))+f^{\prime}(x(s))\right)^{\prime} d s\right]},
$$

and then integrating over $[\delta, t]$ we get

$\ln \left[\frac{\mu+\int_{\beta}^{t} w^{2}(s)\left(f(x(s))+f^{\prime}(x(s))\right)^{\prime} d s}{A}\right] \geq \ln \left(\frac{\left(f(x(\delta))+f^{\prime}(x(\delta))\right)}{f(x(t))+f^{\prime}(x(t))}\right)$, for $t>\delta$.

Thus for every $t>\delta$ it holds that 
$\mu+\int_{\beta}^{t} w^{2}(s)\left(f(x(s))+f^{\prime}(x(s))\right)^{\prime} d s \geq \frac{A\left(f(x(\delta))+f^{\prime}(x(\delta))\right)}{f(x(t))+f^{\prime}(x(t))}$

The last inequality and (2.9) gives

$x^{\prime}(t) \leq-A\left(f(x(\delta))+f^{\prime}(x(\delta))\right)<0$, for all $t>\delta$,

which leads to the contradiction

$\lim _{t \rightarrow \infty} x(t)=-\infty$.

Therefore the theorem implies that the differential equation is oscillatory, and this completes the proof.

\section{Examples}

The following example illustrates the applicability of the theorem.

Consider the second nonlinear order differential equation

$x^{\prime \prime}(t)+\left(x^{\prime}(t)\right)^{2}+e^{t} x^{3}(t)=0$,

for this differential equation we have $f(x)=x^{3}$ and $a(t)=e^{t}$. To show the applicability of Theorem.

For condition (2.1)

$\lim _{t \rightarrow \infty} \inf \int_{\alpha}^{t} a(s) d s=\lim _{t \rightarrow \infty} \inf \int_{\alpha}^{t} e^{s} d s=\lim _{t \rightarrow \infty} \inf \left[e^{s}\right]_{\alpha}^{t}=e^{\alpha}>-\infty$.

For condition $(2.2)$

$$
\begin{aligned}
\lim _{t \rightarrow \infty} \sup \frac{1}{t} \int_{\alpha}^{t}(t-s) a(s) d s & =\lim _{t \rightarrow \infty} \sup \frac{1}{t}\left[(t-s+1) e^{s}\right]_{\alpha}^{t} \\
& =\lim _{t \rightarrow \infty} \sup \left[\frac{e^{t}}{t}-\left(1-\frac{\alpha+1}{t}\right) e^{\alpha}\right]=\lim _{t \rightarrow \infty} \frac{e^{t}}{t}=\infty
\end{aligned}
$$

For condition (2.3) for example put $n=3$

$\lim _{t \rightarrow \infty} \sup \frac{1}{t^{n-1}} \int_{\alpha}^{t}(t-s)^{n-1} a(s) d s=\lim _{t \rightarrow \infty} \sup \frac{1}{t^{2}} \int_{\alpha}^{t}(t-s)^{2} e^{s} d s$

using integration by parts twice we get

$$
\begin{aligned}
\lim _{t \rightarrow \infty} \sup \frac{1}{t^{2}} \int_{\alpha}^{t}(t-s)^{2} e^{s} d s & =\lim _{t \rightarrow \infty} \sup \frac{1}{t^{2}}\left[(t-\alpha)^{2} e^{\alpha}+2(t-\alpha) e^{\alpha}+2\left(e^{t}-e^{\alpha}\right)\right] \\
& =\lim _{t \rightarrow \infty} \sup \left[\begin{array}{c}
\left(1-\frac{\alpha}{t}\right)^{2} e^{\alpha}+2\left(\frac{1}{t}-\frac{\alpha}{t^{2}}\right) e^{\alpha} \\
+\frac{2}{t^{2}}\left(e^{t}-e^{\alpha}\right)
\end{array}\right]=\infty
\end{aligned}
$$

Hence the theorem is applicable.

\section{Acknowledgements}

I would like to extend my thanks to the University of Sharjah for its support. 


\section{References}

[1] Abdullah, H.K.: Oscillation criteria of second order non-linear differential equations, open Journal of Applied Sciences, (2012),vol.12, pp120-122.

[2] Cakmak D.: Oscillation for second order nonlinear differential equations with damping, Dynam. Systems Appl., (2008),vol.17, No.1 pp139-148.

[3] Close W. J.:Oscillation criteria for nonlinear second order equations, Ann. Mat. Pura Appl.,(1969) vol. 82, pp123-134.

[4] Horng J. Li, : Nonoscillatory characterization of a second order linear differential equations, Math. Nacher,(2000), vol.219, pp147-161.

[5] Kim R. J.: Oscillation criteria of differential equations of second order, Korean J. Math., (2011), vol. 19, No.3, pp309-319.

[6] Li W.and Agarwal R. P.: Interval oscillation criteria for second order nonlinear differential equations with damping, compute. Math. Appl., (2000), vol.40, pp217-230.

[7] Sabatini M.: On the period function of $x^{\prime \prime}+f(x)\left(x^{\prime}\right)^{2}+g(x)=0$, J. Differential Equations,(2004) vol.196, pp151-168.

[8] Tyagi J.: An oscillation theorem for a second order nonlinear differential equations with variable potential, Electronic Journal of Differential Equations, vol. (2009), No.19 pp1-5.

[9] Wong J. S. W.: oscillation criteria for second order nonlinear differential equations with integrable coefficients, Proc. Amer. Math.Soc, (1992), vol.115, pp389-395. 